PROCEEDINGS OF THE

AMERICAN MATHEMATICAL SOCIETY

Volume 128, Number 2, Pages 495-501

S 0002-9939(99)05144-8

Article electronically published on June 24, 1999

\title{
A NOTE ON RESTRICTED WEAK-TYPE ESTIMATES FOR BOCHNER-RIESZ OPERATORS WITH NEGATIVE INDEX IN $\mathbb{R}^{n}, n \geq 2$
}

\author{
SUSANA GUTIÉRREZ \\ (Communicated by Christopher D. Sogge)
}

\begin{abstract}
It is shown that the Bochner-Riesz operator on $\mathbb{R}^{n}$ of negative order $\alpha$ is of restricted weak type in the critical points $\left(p_{0}, q_{0}\right)$ and $\left(q_{0}^{\prime}, p_{0}^{\prime}\right)$, where $1 / q_{0}=3 / 4+\alpha / 2, q_{0}=p_{0}^{\prime} / 3$ for $-3 / 2<\alpha<0$ in the two-dimensional case and $1 / q_{0}=(n+1+2 \alpha) / 2 n, q_{0}=(n-1) p_{0}^{\prime} /(n+1)$, for $-(n+1) / 2<$ $\alpha<-1 / 2$ if $n \geq 3$.
\end{abstract}

\section{INTRODUCTION}

The Bochner-Riesz operator $T^{\alpha}$ with index $\alpha$ on $\mathbb{R}^{n}$ is defined on $\mathcal{S}\left(\mathbb{R}^{n}\right)$, the class of rapidly decreasing $\mathcal{C}_{0}^{\infty}$ functions, by

$$
\left(T^{\alpha} f \hat{)}(\xi)=m_{\alpha}(\xi) \widehat{f}(\xi), \quad \xi \in \mathbb{R}^{n},\right.
$$

where $m_{\alpha}(\xi)=\Gamma(\alpha+1)^{-1}\left(1-|\xi|^{2}\right)^{\alpha}$ if $|\xi|<1$ and $m_{\alpha}(\xi)=0$ if $|\xi| \geq 1,{ }^{\wedge}$ denotes the Fourier transform in $\mathbb{R}^{n}$ and $\Gamma$ is the Gamma function.

Considering the inverse Fourier transform of $m_{\alpha}$ which is given by

$$
K_{\alpha}(x)=\int_{\mathbb{R}^{n}} e^{2 \pi i x \xi} m_{\alpha}(\xi) d \xi=\pi^{-\alpha}|x|^{-\frac{n}{2}-\alpha} \mathcal{J}_{\frac{n}{2}+\alpha}(2 \pi|x|),
$$

$T^{\alpha}$ is convolution with the kernel $K_{\alpha}$ above (i.e. $T^{\alpha} f=K_{\alpha} * f$ ). Here $\mathcal{J}_{z}(r)$ denotes the Bessel function of order $z$ (see [W]). We will consider real values of $\alpha$ in the interval $-(n+1) / 2<\alpha<0$.

In order to make an easier description of the results we introduce some notation. For $-(n+1) / 2<\alpha<0$, let

$$
\begin{aligned}
A & =\left(1, \frac{n+1+2 \alpha}{2 n}\right), \quad A=\left(\frac{n-1-2 \alpha}{2 n}, 0\right), \\
B & =\left(\frac{n+1+2 \alpha}{2 n}-\frac{2 \alpha}{n+1}, \frac{n+1+2 \alpha}{2 n}\right), \\
B^{\prime} & =\left(\frac{n-1-2 \alpha}{2 n}, \frac{n-1-2 \alpha}{2 n}+\frac{2 \alpha}{n+1}\right), \\
H & =\left(\frac{1}{2}-\frac{2 \alpha}{n+1}, \frac{1}{2}\right), \quad H^{\prime}=\left(\frac{1}{2}, \frac{1}{2}+\frac{2 \alpha}{n+1}\right),
\end{aligned}
$$

Received by the editors March 29, 1998.

1991 Mathematics Subject Classification. Primary 42B15.

Supported by a grant from Spanish Ministry of Education and Sciences.

(C)1999 American Mathematical Society 


$$
D=\left(\frac{1}{2}-\frac{\alpha}{n+1}, \frac{1}{2}+\frac{\alpha}{n+1}\right), \quad V=(1,0)
$$

and

$$
\begin{aligned}
\Delta_{\alpha}(n)= & \left\{\left(\frac{1}{p}, \frac{1}{q}\right) \in[0,1] \times[0,1] \quad: \quad 0 \leq \frac{1}{q} \leq \frac{1}{p} \leq 1, \quad \frac{1}{q} \leq \frac{1}{p}+\frac{2 \alpha}{n+1},\right. \\
& \left.\frac{1}{q}<\frac{n+1+2 \alpha}{2 n}, \quad \frac{1}{p}>\frac{n-1-2 \alpha}{2 n}\right\} .
\end{aligned}
$$

Then $\Delta_{\alpha}(n)$ is the region in the plane which consists of the closed solid pentagon $A B B^{\prime} A^{\prime} V$ except for the closed segments $A B$ and $A^{\prime} B^{\prime}$ (see Figure 1). Observe that the point $B$ lies on the line $1 / q=(n+1)(1-1 / p) /(n-1)$.

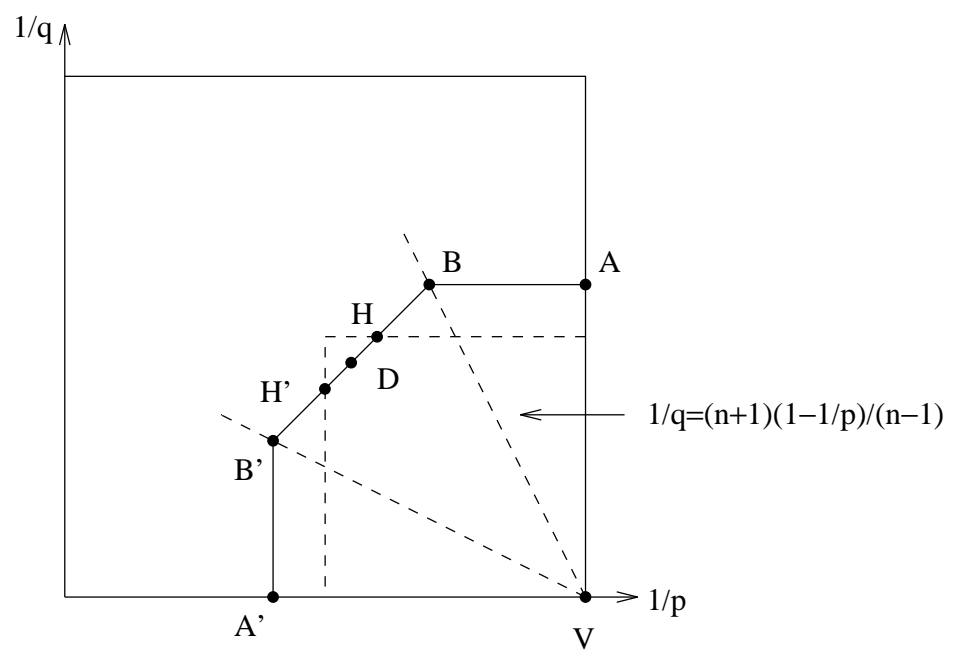

FiguRE 1. Region $\Delta_{\alpha}(n)$

It is well known that $(1 / p, 1 / q)$ being in $\Delta_{\alpha}(n)$ is a necessary condition for finding strong type $(p, q)$-boundedness of $T^{\alpha}$ (see [Bo], [CSr]). Some partial results are also known for the converse statement to be true. Background related to the subject can be found in $[\mathrm{Br}],[\mathrm{Sg}],[\mathrm{CSr}],[\mathrm{BMO}]$ and $[\mathrm{Bk}]$. In particular the problem of $T^{\alpha}$ being bounded, as an operator defined from $L^{p}$ to $L^{q}$, has been completed for values of the $\alpha$-parameter in the interval $[-(n+1) / 2,-1 / 2]$ in all dimensions (see $[\mathrm{BMO}]$ ), and recently Bak (see [Bk]) extended the result for $-3 / 2<\alpha<0$ in the two-dimensional case.

On the other hand, some results have been recently proved (see $[\mathrm{Bk}]$ ) concerning the problem of finding weak-type estimates for this kind of operator on the boundary of the region $\Delta_{\alpha}(n)$ corresponding with the closed upper segment $A B$. In this sense no positive result has been established for the weak boundedness of $T^{\alpha}$ in the "critical points" $B$ and $B^{\prime}$.

The main goal of this paper is to establish restricted weak-type estimates for Bochner-Riesz means of negative order in these two extreme points.

As a consequence, using an interpolation argument, we will recover strong-type boundedness of $T^{\alpha}$ and boundedness properties of such operators in Lorentz spaces. 
We will prove the following theorem in the next section.

Theorem 1. Let $T^{\alpha}$ be the Bochner-Riesz operator of order $\alpha$ on $\mathbb{R}^{n}$. If

(i) $\frac{1}{q}=\frac{n+1+2 \alpha}{2 n} \quad$ and $\quad \frac{1}{p}=\frac{n+1+2 \alpha}{2 n}-\frac{2 \alpha}{n+1}$, or

(ii) $\frac{1}{p}=\frac{n-1-2 \alpha}{2 n} \quad$ and $\quad \frac{1}{q}=\frac{n-1-2 \alpha}{2 n}+\frac{2 \alpha}{n+1}$,

then there exists a constant $C=C(p, q, \alpha)$ such that

$$
\left\|T^{\alpha} f\right\|_{q, \infty} \leq C\|f\|_{p, 1}
$$

whenever $-3 / 2<\alpha<0$ in the two-dimensional case and $-(n+1) / 2<\alpha<-1 / 2$ in higher dimensions.

The following remarks are direct consequences of the restricted weak-type estimates given in the previous theorem, known boundedness properties of $T^{\alpha}$, deduced from the fact that $T^{\alpha}$ is pointwise bounded by a fractional integral of order $(n-1) / 2-\alpha$, and real interpolation arguments (see [StWe], [BeJ] and [BSh]).

Remark 1. Restricted weak-type estimates in $B$ and $B^{\prime}$ lead us to recover the optimal scheme for strong $(p, q)$-boundedness of $T^{\alpha}$ : if either $n=2$ and $-3 / 2<$ $\alpha<0$, or $n \geq 3$ and $-(n+1) / 2<\alpha<-1 / 2$, then $T^{\alpha}$ is of strong type $(p, q)$ if and only if $(1 / p, 1 / q) \in \Delta_{\alpha}(n)$ (see also [BMO] and $\left.[\mathrm{Bk}]\right)$.

Remark 2. An analogous result for values of $\alpha$ such that $-1 / 2 \leq \alpha<0$ and $n \geq 3$ would imply the optimal scheme for $T^{\alpha}$ to be a strong type $(p, q)$-operator which is still unknown except for the case $\alpha=-1 / 2$.

Remark 3. As $T^{\alpha}$ is of weak type $(1,2 n / n+1+2 \alpha)$ (i.e. in $A$ ), we conclude that for $1 / q=(n+1+2 \alpha) / 2 n,(n+1+2 \alpha) / 2 n-2 \alpha /(n+1)<1 / p \leq 1$ and if either $n=2$ and $-3 / 2<\alpha<0$, or $n \geq 3$ and $-(n+1) / 2<\alpha<-1 / 2$, then $T^{\alpha}$ is of weak type $(p, q)$ (see also $[\mathrm{Bk}])$.

Remark 4. Finally the restricted weak-type estimate in $B^{\prime}$ and the boundedness of $T^{\alpha}$ from $L^{p}, 1 / p=(n-1-2 \alpha) / 2 n$, to BMO yield the following estimates on the left closed segment $A^{\prime} B^{\prime}$ of the set $\Delta_{\alpha}(n)$ : if either $n=2$ and $-3 / 2<\alpha<0$, or $n \geq 3$ and $-(n+1) / 2<\alpha<-1 / 2$, then

$$
\left\|T^{\alpha} f\right\|_{p, r} \leq C\|f\|_{q}
$$

where $1 / p=(n-1-2 \alpha) / 2 n, 0<1 / q<(n-1-2 \alpha) / 2 n+2 \alpha /(n+1)$ and $1 / r=(n+1)(1 / q) /(n-1)+(n-1-2 \alpha) / 2 n$.

Note that $T^{\alpha}$ cannot be a weak operator on the closed segment $A^{\prime} B^{\prime}$. Indeed, a duality argument would imply that $T^{\alpha}$ is bounded from the Lorentz space $L^{q, 1}$ to $L^{p}$ with $(1 / p, 1 / q)$ lying on the closed upper segment $A B$, however the counterexample for the strong type boundedness of $T^{\alpha}$ on this segment is given by the characteristic function of a ball (see [Br, p.231]).

Remark 5. The line $1 / q=(n+1)(1-1 / p) /(n-1)$ is critical in the sense that finding estimates for $T^{\alpha}$ in the point $B$ implies, by duality and interpolation, obtaining sharp estimates for $T^{\alpha}$ in points $(1 / p, 1 / q)$ in the closed pentagon $A B B^{\prime} A^{\prime} V$. 
Remark 6 . For the sake of completeness we summarize what is known for $-1 / 2 \leq$ $\alpha<0, n \geq 3$ (see [BMO] and [Bk]):

(a) $T^{\alpha}$ is of weak-type $(p, q)$ for $1 / q=(n+1+2 \alpha) / 2 n$ if either

$$
\begin{gathered}
\alpha=-1 / 2, \quad \frac{n+3}{2(n+1)}<\frac{1}{p} \leq 1, \quad \text { or } \\
-1 / 2<\alpha<0, \quad \frac{n+3}{2(n+1)} \leq \frac{1}{p} \leq 1, \quad \text { and }
\end{gathered}
$$

(b) $T^{\alpha}$ is of strong type $(p, q)$ for $1 / q-1 / p=2 \alpha /(n+1)$ if either

$$
\begin{gathered}
\left.\alpha=-1 / 2, \quad \frac{1}{2}<\frac{1}{p}<\frac{n+3}{2(n+1)} \quad \text { (i.e. open seg. } B B^{\prime}\right) \quad \text { or } \\
\left.-1 / 2<\alpha<0, \quad \frac{1}{2} \leq \frac{1}{p} \leq \frac{1}{2}-\frac{2 \alpha}{n+1} \quad \text { (i.e. closed seg. } H H^{\prime}\right) .
\end{gathered}
$$

Case (a) follows by choosing $\left(p_{0}, q_{0}\right)=(p, p),\left(p_{1}, q_{1}\right)=\left(p,(n-1) p^{\prime} /(n+1)\right)$ in (2) and replacing these values in the proof of the theorem below (this is the argument in $[\mathrm{Bk}])$.

A bootstrap argument and the known boundedness of $T^{\alpha}$ in the duality line (see [St, pg.374], [T1], [T2]) yield:

$$
\begin{aligned}
\left\|T^{\alpha} f\right\|_{2}^{2} & =\int T^{\alpha} f \overline{T^{\alpha} f} d x=\int m_{\alpha} \widehat{f} \overline{m_{\alpha} \widehat{f}} d \xi=\Gamma(\alpha+1)^{-2} \Gamma(2 \alpha+1) \int f \overline{T^{2 \alpha} f} d x \\
& \leq C_{\alpha}\|f\|_{p}\left\|T^{2 \alpha} f\right\|_{p^{\prime}} \leq C_{\alpha}\|f\|_{p}^{2},
\end{aligned}
$$

for $1 / p=1 / 2-2 \alpha /(n+1)$ and $-1 / 2<\alpha<0$. Then, duality and interpolation conclude case (b) for $-1 / 2<\alpha<0$. Case $\alpha=-1 / 2$ in (b) was established in $[\mathrm{BMO}]$ using an analytic interpolation argument.

The proof of the theorem is based on the following well-known estimates for the dyadic operators associated to $T^{\alpha}\left(T^{\alpha}=K_{\alpha} * f\right)$ :

- [Sg, Lemma 5.3] If $n=2,1 / q=3(1-1 / p)$ and $1 / q<3 / 4$,

$$
\left\|K_{\alpha}^{j} * f\right\|_{L^{q}} \leq C_{p, q} 2^{-\left(\frac{3}{2}+\alpha\right) j+\frac{2 j}{q}}\|f\|_{L^{p}} .
$$

- [CSr, Lemma 5.1] If $n \geq 3,(n+1)(1-1 / p) /(n-1) \leq 1 / q \leq 1 / p,(n+3) /$ $2(n+1) \leq 1 / p \leq 1$,

$$
\left\|K_{\alpha}^{j} * f\right\|_{L^{q}} \leq C_{p, q} 2^{-\alpha j} 2^{-\frac{j}{2}} 2^{j n\left(\frac{1}{q}-\frac{1}{2}\right)}\|f\|_{L^{p}},
$$

where $K_{\alpha}^{j}, j=1,2, \ldots$, arises by decomposing dyadically the kernel $K_{\alpha}$, that is, $K_{\alpha}^{j}(x)=K_{\alpha}(x) \psi\left(x / 2^{j}\right)$ with $\psi$ smooth of compact support in an annulus.

In order to obtain the desired result in the critical points $B$ and $B^{\prime}$ we will just make use of those estimates in (1) and (2) related to the critical line $1 / q=$ $(n+1)(1-1 / p) /(n-1)$.

\section{Proof of the theorem}

We will prove the restricted weak-type estimate

$$
\left\|T^{\alpha} f\right\|_{q, \infty} \leq C\|f\|_{p, 1},
$$

for $p$ and $q$ in the case (i). Case (ii) in the theorem is proved considering the dual estimates for those given in (1) and (2) using the same arguments. 
It is known that estimate (3) is equivalent to the Lorentz space estimate,

$$
\left\|T^{\alpha} \chi_{\Omega}\right\|_{q, \infty} \leq C\left\|\chi_{\Omega}\right\|_{p}
$$

that is,

$$
\left|\left\{x \in \mathbb{R}^{n} \quad: \quad\left|T^{\alpha} \chi_{\Omega}(x)\right|>\lambda\right\}\right| \leq C\left(\frac{|\Omega|^{\frac{1}{p}}}{\lambda}\right)^{q}, \quad \forall \lambda>0,
$$

for the same $p$ and $q$ (see [StWe, pg.195 t3.13]) where $\chi_{\Omega}$ denotes the characteristic function of a finite measured set $\Omega$ and $|\cdot|$ denotes Lebesgue measure.

It is enough to estimate the level set, $A$, defined by

$$
A=\left\{x \in \mathbb{R}^{n} \quad: \quad \Re e T^{\alpha} f(x)>\lambda\right\}, \quad \lambda>0 .
$$

Let $B_{R}$ be a ball centered in the origin of a fixed radius $R$ to be determined in what follows. Then, we can write

$$
\begin{aligned}
|A| & =\int_{A} d x \leq \frac{1}{\lambda} \Re e \int_{A} T^{\alpha} f(x) d x=\frac{1}{\lambda} \Re e \int K_{\alpha} * f(x) \chi_{A}(x) d x \\
& =\frac{1}{\lambda} \Re e\left\{\int K_{\alpha} \chi_{B_{R}} * f(x) \chi_{A}(x) d x+\int K_{\alpha} \chi_{B_{R}} * f(x) \chi_{A}(x) d x\right\} \\
& =\frac{1}{\lambda} \Re e\{I+I I\} .
\end{aligned}
$$

Here $B_{R}{ }^{c}$ is the complementary of the ball $B_{R}$ in $\mathbb{R}^{n}$.

In order to make use of the estimates for the dyadic operators associated with the kernel $T^{\alpha}$, we first consider a dyadic decomposition of $\mathbb{R}^{n}$ in the usual way.

From the non-singular behaviour of the kernel $K_{\alpha}$ in a neighbourhood of the origin and the estimates in (1) and (2) with exponent $q_{0}=2\left(1 / q_{0}=3 / 4-\varepsilon\right.$ if $n=2)$ and $1 / p_{0}=(n+3) / 2(n+1)$ (resp. $\left.1 / p_{0}=3 / 4+\varepsilon / 3\right)$, it follows easily that

$$
\begin{aligned}
\left\|K_{\alpha} \chi_{B_{R}} * f\right\|_{q_{0}} & \leq C \sum_{j=0}^{\log _{2} R}\left\|K_{\alpha}^{j} * f\right\|_{q_{0}} \leq C\left(\sum_{j=0}^{\log _{2} R} 2^{-\alpha j} 2^{-\frac{j}{2}} 2^{j n\left(\frac{1}{q_{0}}-\frac{1}{2}\right)}\right)\|f\|_{p_{0}} \\
& \leq C R^{\frac{n}{q_{0}}-\frac{n+1+2 \alpha}{2}}\|f\|_{p_{0}}
\end{aligned}
$$

where $-(n+1) / 2<\alpha<n / q_{0}-(n+1) / 2\left(\right.$ resp. $\left.-3 / 2<\alpha<2 / q_{0}-3 / 2\right)$ so that the power of $2^{j}$ in the latter series is positive.

On the other hand using the same estimates with indexes $q_{1}=\infty$ and $p_{1}=1$ we obtain that

$$
\begin{aligned}
\left\|K_{\alpha} \chi_{B_{R}^{c}} * f\right\|_{q_{1}} & \leq C \sum_{j=\log _{2} R}^{\infty}\left\|K_{\alpha}^{j} * f\right\|_{q_{1}} \leq C\left(\sum_{j=\log _{2} R}^{\infty} 2^{-j\left(\frac{n+1}{2}+\alpha\right)}\right)\|f\|_{p_{1}} \\
& \leq C R^{-\frac{n+1}{2}-\alpha}\|f\|_{p_{1}}
\end{aligned}
$$

because of the convergence of the truncated series $\forall \alpha>-(n+1) / 2$ and $n \geq 2$.

Then, Hölder's inequality yields

$$
\begin{aligned}
|I| & \leq \int\left|K_{\alpha} \chi_{B_{R}} * f\right| \chi_{A}(x) d x \leq\left\|K_{\alpha} \chi_{B_{R}} * f\right\|_{q_{0}}\left\|\chi_{A}\right\|_{q_{0}^{\prime}} \\
& \leq C R^{\frac{n}{q_{0}}-\frac{n+1+2 \alpha}{2}}\|f\|_{p_{0}}|A|^{\frac{1}{q_{0}^{\prime}}}
\end{aligned}
$$


and

$$
\begin{aligned}
|I I| & \leq \int\left|K_{\alpha} \chi_{B_{R}^{c}} * f(x)\right| \chi_{A}(x) d x \leq\left\|K_{\alpha} \chi_{B_{R}^{c}} * f\right\|_{q_{1}}\left\|\chi_{A}\right\|_{q_{1}^{\prime}} \\
& \leq C R^{-\frac{n+1}{2}-\alpha}\|f\|_{1}|A| .
\end{aligned}
$$

By (4), (5) and (6) we conclude

$$
|A| \leq \frac{C}{\lambda}\left\{R^{\frac{n}{q_{0}}-\frac{n+1+2 \alpha}{2}}\|f\|_{p_{0}}|A|^{\frac{1}{q_{0}^{\prime}}}+R^{-\frac{n+1}{2}-\alpha}\|f\|_{1}|A|\right\} .
$$

Now from the above estimate applied to the characteristic function $f=\chi_{\Omega}$, since $\Omega$ is a finite measured set in $\mathbb{R}^{n}$, and $R=|\Omega|^{\frac{q_{0}}{n p_{0}^{\prime}}}|A|^{\frac{1}{n}}$, so that the summands above are equal, we conclude

$$
\begin{aligned}
|A| & \leq \frac{C}{\lambda}\left\{R^{\frac{n}{q_{0}}-\frac{n+1+2 \alpha}{2}}|\Omega|^{\frac{1}{p_{0}}}|A|^{\frac{1}{q_{0}^{\prime}}}+R^{-\frac{n+1}{2}-\alpha}|\Omega||A|\right\} \\
& \leq \frac{C}{\lambda}|\Omega|^{1-\frac{q_{0}}{p_{0}^{\prime}}\left(\frac{n+1+2 \alpha}{2 n}\right)}|A|^{1-\frac{n+1+2 \alpha}{2 n}}
\end{aligned}
$$

and therefore

$$
|A| \leq C\left(\frac{|\Omega|^{1-\frac{q_{0}}{p_{0}^{\prime}}\left(\frac{n+1+2 \alpha}{2 n}\right)}}{\lambda}\right)^{\frac{\frac{1}{n+1+2 \alpha}}{2 n}}, \quad \forall \lambda>0 .
$$

The result follows by replacing the corresponding values of $p_{0}, q_{0}$ and $\alpha$ depending on the considered dimension.

\section{ACKNOWLEDGMENTS}

I would like to thank L.Vega for suggesting this problem to me, and for his continued ideas and patient help.

\section{REFERENCES}

[BSh] C. Bennet \& R. Sharpley, Interpolation of operators, Academic Press, Inc., Orlando, Florida, (1988).

[BeJ] J. Bergh \& J. Löfström, Interpolation of spaces, An introduction, Springer-Verlag, BerlinHeidelberg-New York, (1976).

[Bo] J. Bourgain, Besicovitch-type maximal operators and applications to Fourier analysis, Geom. and Funct. Anal., 1, (1991), no.2, 147-187. MR 92g:42010

[Bk] J.-G Bak, Sharp estimates for the Bochner-Riesz operator of negative order in $\mathbb{R}^{2}$, Proc. Amer. Math. Soc., 125, (1997), no. 7, 1977-1986. MR 97i:42011

[BMO] J.-G. Bak, D. McMichael \& D. Oberlin, $L^{p}-L^{q}$ Estimates off the line of duality, J. Austral. Math. Soc., 58, (1995), 154-166.

[Br] L. Börjeson, Estimates for the Bochner-Riesz operator with negative index, Indiana U. Math. J., 35, (1986), no.2, 225-233. MR 87f:42036

[CSr] A. Carbery \& F. Soria, Almost-everywhere convergence of Fourier integrals for functions in Sobolev spaces, and an $L^{2}$-localisation principle, Rev. Mat. Iberoamericana, 4, (1988), no.2, 319-337. MR 91d:42015

[Sg] C. Sogge, Oscillatory integrals and spherical harmonics, Duke Math. J., 53, (1986), no.1, 43-65. MR 91d:35037

[St] E.M. Stein, Harmonic analysis: Real-variable methods, orthogonality, and oscillatory integrals, Princeton U. Press, Princeton, New Jersey, (1993). MR 95c:42002

[StWe] E.M. Stein \& G.Weiss, An introduction to Fourier analysis on Euclidean spaces, Princeton U. Press, Princeton, New Jersey, 1975.

[T1] P. Tomas, A restriction theorem for Fourier transform, Bull. Amer. Math. Soc., 81, (1975), no.2, 477-478. MR 50:10681 
[T2] P.A. Tomas, Restriction theorems for the Fourier transform, Harmonic Analysis in Euclidean Spaces (2 volumes), G. Weiss and W.Wainger (eds.), Proc. Symp. Pure Math. \# 35, American Math. Society, (1979), part 1, 111-114. MR 81d:42029

[W] G.W. Watson, Theory of Bessel functions, Cambridge University Press, Cambridge, 1944. MR 6:64a

Departamento de Matemáticas, Facultad de Ciencias, Universidad del País Vasco (UPV-EHU), Aptdo 644, 48080 Bilbao, Spain

E-mail address: mtbgugrs@lg.ehu.es 